\title{
Neurological Consequences Stemming From Pulmonary Alveolar Proteinosis and Opportunistic Infections
}

Kirshner B*, Franco T and Dean R

Graduate Medical Education, Coliseum Medical Center, Macon, Georiga, USA

\begin{abstract}
Pulmonary alveolar proteinosis (PAP) is a rare lung disease in which macrophage dysfunction causes a nonproductive cough and progressive dyspnea. However, mortality in roughly $20 \%$ of these patients is due to opportunistic infections by fungal pathogens, Nocardia spp, and mycobacteria. In this case, we discuss a 52 -year-old African American male who presented with a month-long history of progressively worsening dyspnea and frequent nonproductive cough. The patient's medical history was significant for a diagnosis of cavitary pneumonia one year ago. He was treated for community acquired pneumonia. A week later, the patient presented back to the emergency room with seizures and an altered mental status with a lesion appearing on head CT. The brain lesion was removed and tested positive for Nocardia. However, the diagnosis of PAP was not considered at that time. This case is significant because PAP should be considered as a differential when a patient presents with dyspnea, cough, and opportunistic infection. If the patient was diagnosed with PAP at his initial visit, the Nocardia infection could have been found and treated conservatively before it progressed to seizures and an emergent surgery.
\end{abstract}

Keywords: Opportunistic infection; Dyspnea; Lung pathology; Microbiology; Pulmonary alveolar proteinosis; Seizure; Bronchial lavage

\section{Case Report}

A 52 year old African American male presented to the Emergency room with a month long history of worsening dyspnea as well as a frequent non-productive cough. He described his dyspnea as a gradual state, explaining it originally presented with exertion but had recently progressed to while he was at rest. The patient denied any other symptoms associated with the shortness of breath, specifically denying any chest pain, subjective fevers, or generalized weakness.

The patient has a significant pulmonary past medical history with a cavitary pneumonia diagnosed one year prior in the patient's upper right lobe. During his hospital stay one year ago, tuberculosis testing was negative and he was treated with the proper antibiotics for a community acquired pneumonia. A week later, the patient presented back to the Emergency Room with seizures and an altered mental status. On head CT he was found to have a lesion in the left temporal lobe of his brain. On surgical evacuation, cultures were sent of the lesion and returned positive for Nocardia species.

On this admission, physical exam revealed a patient in no acute distress with mild dyspnea. Cardiac, abdominal, neurological and pulmonary exams were benign with only diffuse mild expiratory wheezing throughout the lung fields. Laboratory data revealed Haemoglobin of $11.2 \mathrm{G} / \mathrm{dL}$, White Blood Cells of $7.0 \mathrm{~K} / \mathrm{uL}$, and an unremarkable chemistry panel. His Arterial Blood Gas on room air was as follows; $\mathrm{pH}$ of 7.44, 02 saturation of 85.0, $\mathrm{pCO}_{2}$ of 30.0, a $\mathrm{pO}_{2}$ of 47 , and $\mathrm{HCO}_{3}$ of 20.3 .

Chest radiograph revealed chronic changes consistent with a previous right upper lobe lesion with scarring, as well as diffuse reticulonodular densities throughout both lung fields (Figure 1). Follow up Chest CT revealed diffuse ground glass infiltrates throughout both lungs as well as slightly nodular patchy infiltrates in the upper lobes bilaterally (Figure 2).

Upon a pathology workup of the patient's bronchial washing, the gross specimen consisted of a white, cloudly material with a WBC of $4874 \mathrm{u} / \mathrm{L}$ (reference range below 300), Lymphocytes of 26\% (reference range $3 \%$ to $4 \%$ ) and Macrophages of "Low to Moderate" (reference

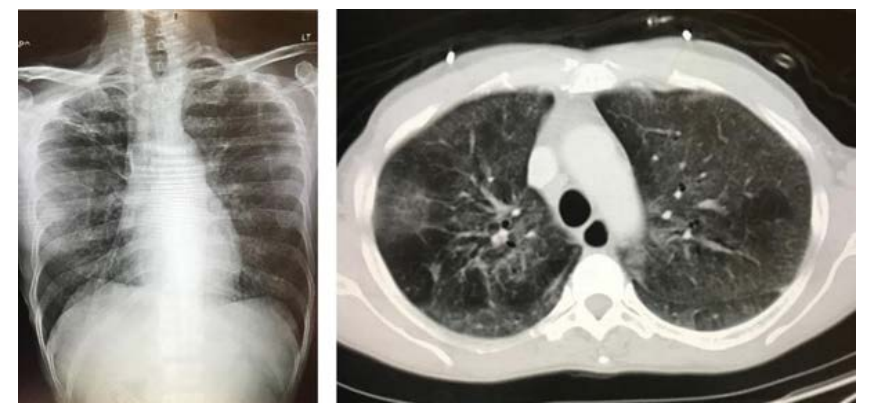

Figure 1: Chest radiograph.

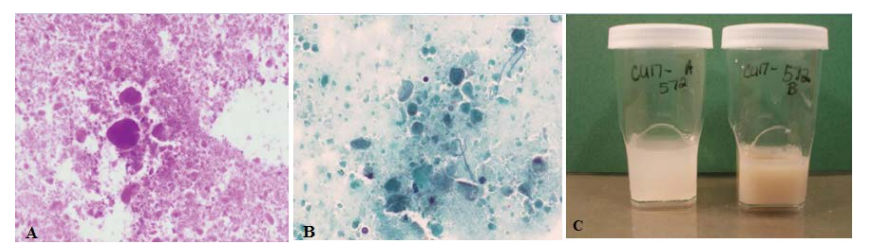

Figure 2: Chest CT.

range $95 \%$ to $96 \%$ ). On microscopy, the sample yielded moderate cellularity with scattered lymphocytes, foamy macrophages containing eosinophilic granules, and diffuse extracellular protienacious globular material which stained strongly positive with a periodic acid schiff(PAS) stain, as well as dense globules that were also seen with Papanicolaou (PAP) stain. Congo red stain was used to rule out amloydosis.

${ }^{*}$ Corresponding author: Brandon Kirshner, Graduate Medical Education Coliseum Medical Center, Macon, Georiga, USA, Phone: 585-857-6975; E-mail: Brandon.Kirshner@hcahealthcare.com

Received June 06, 2018; Accepted June 11, 2018; Published June 17, 2018

Citation: Kirshner B, Franco T, Dean R (2018) Neurological Consequences Stemming From Pulmonary Alveolar Proteinosis and Opportunistic Infections. J Pulm Respir Med 8: 459. doi: 10.4172/2161-105X.10004597

Copyright: (c) 2018 Kirshner B, et al. This is an open-access article distributed under the terms of the Creative Commons Attribution License, which permits unrestricted use, distribution, and reproduction in any medium, provided the original author and source are credited. 
On further laboratory testing, the patient was found to have a Lactate Dehydrogenase of $579 \mathrm{U} / \mathrm{L}$, an IgG of $1874 \mathrm{mg} / \mathrm{dL}$, a negative ANA, and Granulocyte macrophage colony stimulating factor antibodies of $1.9 \mathrm{pg} / \mathrm{mL}$ (normal range below $0.5 \mathrm{pg} / \mathrm{mL}$ ). The patient's clinical findings, laboratory data, and bronchial washing microscopy led to the diagnosis of Pulmonary Alveolar Proteinosis. After diagnosis, the patient was transferred to a larger institution and underwent a whole lung lavage as treatment. Per their records, the patient tolerated the procedure well and was instructed to follow up in six months for a repeat treatment.

Unfortunately for the patient, he returned to the emergency room with intractable seizures likely due to the post-surgical changes from the Nocardia infection evacuation one year prior. Failing conservative medical management, the patient had to be intubated and sedated with propofol to halt his seizure activity. He was stabilized and transferred to a nearby center with an appropriate neurological ICU. Due to his prolonged seizure activity stemming from physical foci, his prognosis was poor at best.

\section{Discussion}

Pulmonary alveolar proteinosis (PAP) is a rare disease with an estimated annual incidence of 0.36 cases per million [1]. PAP is asymptomatic in roughly one-third of cases. The typical symptoms are non-productive cough and progressive dyspnea [2]. The first reported case of PAP was in 1958 where it was described as a condition where alveoli were filled with periodic acid-Schiff (PAS)-positive lipoproteinaceous material [3]. The lipoproteinaceous material was later found to be composed of pulmonary surfactant and cellular debris [3]. Surfactant is made and secreted by type II alveolar epithelial cells and subsequently removed and recycled by alveolar macrophages. The pathology of PAP is attributed to problems with pulmonary surfactant clearance by alveolar macrophages.

There are three types of PAP. Hereditary PAP causes less than $1 \%$ of PAP cases. Hereditary PAP is due to mutations in CSF2RA or $C S F 2 R B$ which disrupts GM-CSF signalling $[1,2]$. Secondary PAP is associated with toxic environmental exposures, chronic inflammation and infection, immune deficiency, cancer, and hematologic disease. The autoimmune type is responsible for $90 \%$ of PAP cases. Autoimmune PAP is associated with a high level of anti-GM-CSF autoantibody which disrupts GM-CSF signaling which adversely affects the activation and function of immune cells, alveolar macrophages included [1,2]. The patient in this case report was found to have BAL findings of milky white fluid, cytopathology which showed macrophages filled with eosinophilic granules and diffuse extracellular PAS+ proteinaceous globules, and high levels of GM-CSF autoantibodies. Elevated levels of GM-CSF autoantibodies are 100\% sensitive and specific for autoimmune PAP [2].

Patients with PAP are known to be at an increased risk of rare, opportunistic infections due to fungal pathogens, Nocardia spp, and mycobacteria [4,5]. Opportunistic infections account for roughly $20 \%$ of deaths due to PAP [1]. A literature review done by Punatar et al. identified seventy-five reported cases of PAP and opportunistic infection from 1950 to July of 2010. Of those seventy-five cases, thirtytwo $(43 \%)$ patients had Nocardia infection, twenty-eight $(37 \%)$ had mycobacterial infection and fifteen (20\%) had fungal infections [6]. The lungs were the most common site of infection, however extrapulmonary infection occurred in twenty-seven (32\%) cases. Brain involvement was noted in six of the thirty-two patients with Nocardia spp infection [6]. The susceptibility of patients with PAP for infected with these rare, opportunistic pathogens is believed to be due to disruption of GM-CSF signaling and priming of neutrophils due destruction of GM-CSF by the autoantibodies present in PAP [5,7] The disseminated, extra-pulmonary involvement seen in $32 \%$ of these cases implies that the disruption of GM-CSF signaling seen in PAP is not just localized within the lungs but is systemic $[1,6]$.

The significance of GM-CSF autoantibodies in PAP was first discovered in 1994 when GM-CSF -/-mice were created that presented with similar histopathologic features as patients with PAP [7]. GMCSF potentiates macrophage and neutrophil antimicrobial activity by increasing $\mathrm{CD} 1 \mathrm{~b}$ adhesion molecule which promotes adhesion and recruitment into infected tissues. GM-CSF also augments phagocytosis and oxidative burst in neutrophils [8]. Neutrophils from patients with PAP have a similar ultrastructure when compared to controls. Neutrophil counts are also normal. However, the neutrophil function is severely impaired. Cellular adhesion, oxidative burst and bacterial killing were reduced in patients with PAP compared to the control group [4]. Additionally, the proportion of phagocytic neutrophils in whole blood and the number of phagocytosed vesicles per neutrophil were likewise reduced [4]. These problems with neutrophil function account for the increased risk of opportunistic infection associated with PAP.

Nocardia is one of the most reported infections associated with PAP [6]. This is because GM-CSF production appears to be a critical part of the immune system's response to Nocardia. Nocardia-induced phosphorylation of pSTAT [4] in the production pathway of GMCSF was seen in vitro 1.5 hours after incubation of PBMCs with live Nocardia [9]. This rapid time period is consistent with the time required for GM-CSF synthesis.

This case report is significant because it highlights the importance of identifying PAP and GM-CSF autoantibodies as a potential cause of unexplained opportunistic infection. In a review of 75 patients, PAP was the initial diagnosis of only 19 patients. An opportunistic infection diagnosis preceded the underlying PAP diagnosis in 23 patients with a mean of a 17-month difference between the two diagnoses [4]. In the other cases, the timeline of diagnoses was not clear. Current research shows that GM-CSF infusion has great possibility as a potential therapeutic intervention. In one particular study, GM-CSF infusion was given over 72 hours to 40 adult patients with sepsis due to infection. GM-CSF infusion caused the upregulation of CD11b on circulating neutrophils and monocytes within the infusion group. Mortality and organ failure in the infusion group versus the control group were similar but the infection cleared appreciably in the infusion group [10]

\section{References}

1. Seymour J F, Presneill JJ (2002) Pulmonary alveolar proteinosis: Progress in the first 44 years. Am J Respire Crit Car Med 166: 215-235.

2. Suzuki T, Trapnell BC (2016) Pulmonary alveolar proteinosis syndrome. Clin Chest Med 37: 431-440.

3. Rosen SH, Castleman B, Liebow AA (1958) Pulmonary alveolar proteinosis. N Engl J Med 258: 1123-1142.

4. Uchida K, Beck DC, Yamamoto T, Berclaz PY, Abe S, et al. (2007) GM-CSF autoantibodies and neutrophil dysfunction in pulmonary alveolar proteinosis. $\mathrm{N}$ Engl J Med 356: 567-579.

5. Kitamura T, Tanaka N, Watanabe J, Yamada Y, Kanegasaki S, et al. (1999) Idiopathic pulmonary alveolar proteinosis as an autoimmune disease with neutralizing antibody against granulocyte/macrophage colony stimulating factor. J Exp Med 190: 875-880.

6. Punatar AD, Kusne S, Blair JE, Seville MT, Vikram HR, et al. (2012) Opportunistic infections in patients with pulmonary alveolar proteinosis. Journal of Infection 65: 173-179.

7. Stanley E, Lieschke GJ, Grail D, Metcalf D, Hodgson G, et al. (1994) Granulocyte/macrophage colony-stimulating factor-deficient mice show no 
Citation: Kirshner B, Franco T, Dean R (2018) Identification of Two Novel Regulatory Elements in the IL2RA Gene Locus. J Pulm Respir Med 8: 459. doi: 10.4172/2161-105X.1000459

major perturbation of hematopoiesis but develop a characteristic pulmonary pathology. Proc Natl Acad Sci USA 91: 5592-5596.

8. Fleischmann J, Golde DW, Wiesbart RH, Gasson JC (1986) Granulocytemacrophage colony stimulating factor enhances phagocytosis fo bacteria by human neutrophils. Blood 68: 708-711.

9. Rosen LB, Nuno RP, Figueiredo C, Fiske LC, Ressner RA, et al. (2015)
Nocardia-Induced Granulocyte macrophage Colony-Stimulating Factor is Neutralized by Autoantibodies in Disseminated/Extrapulmonary Nocardiosis. Clin Infect Dis 60: 1017-1025.

10. Rosenbloom AJ, Linden PK, Dorrance A, Penkosky N, Cohen-Melamed MH , et al. (2005) Effect of granulocyte-monocyte colony-stimulating factor therapy on leukocyte function and clearance of serious infection in nonneutropenic patients. Chest 127: 2139-2150. 